\title{
Maxwell-Systems with Nonlinear Polarization
}

\author{
H.T. Banks \\ Center for Research in Scientific Computation \\ North Carolina State University \\ Raleigh, NC 27965-8205 \\ Gabriella A. Pinter \\ Department of Mathematical Sciences \\ University of Wisconsin Milwaukee \\ Milwaukee, WI 53201-0413
}

March 15, 2002

\begin{abstract}
We establish well-posedness results for a model describing the propagation of high-intensity electromagnetic waves in a nonlinear medium. The nonlinear material properties are represented by a nonlinear polarization in the form of a convolution. We also include some remarks on potential applications.
\end{abstract}

\section{Introduction}

In this paper we consider a time-domain model for the propagation of high-intensity electromagnetic waves (e.g., laser beams) in dielectric materials. This work is a continuation of the efforts in the monograph [1], where the authors investigated a similar model using the full Maxwell's equations together with linear constitutive relations in a variational approach for the propagation of microwaves through dielectric layers. The variational approach facilitates the incorporation of antenna sources in the model and provides an approximate computation of the electromagnetic transients like the Sommerfeld and Brillouin precursors. The monograph [1] presents a one-dimensional model of planar pulsed microwaves incident on a slab 
of material and through studies of the forward and inverse problems provides a rigorous theoretical and computational basis for potential new electromagnetic interrogation techniques. Our goal in this paper is to broaden the applicability of the results in [1] in the following sense: the model in the monograph contains a linear constitutive relationship describing material polarization in a convolution representation. Such a formulation includes Debye and Lorentz polarization models and is adequate to describe the interaction of microwaves with dielectric materials. However, the polarization mechanism cannot be assumed to be linear in the case of very high intensity electromagnetic waves, like lasers. Thus in this paper we develop theoretical results using a representation of the polarization by a nonlinear convolution. In particular, we take a physical model similar to that in [1], introduce a polarization law

$$
P(t, z)=\int_{0}^{t} g(t-s, z)(E(s, z)+f(E(s, z))) d s,
$$

and establish the well-posedness of the resulting system. This formulation can be interpreted as a generalization of the Debye or Lorentz polarization models in the sense that the polarization dynamics is driven by a nonlinear function of the electric field. We show the global existence-uniqueness and continuous dependence on data of weak solutions under general assumptions on the nonlinearity $f$. These conditions are satisfied by a number of locally polynomial nonlinearities proposed in the nonlinear optics literature (e.g., see the "second order" optical models involving cubic nonlinearities in $[6,7]$.) The analysis uses techniques similar to those in [2] with the added difficulty of treating the terms that result from the boundary conditions. Based on the theoretical results, rigorous computational methods can be developed for the forward problem and subsequently for the associated inverse problems.

The literature on nonlinear optics and Maxwell's equations is, of course, vast. Good introductions to nonlinear optics can be found in $[6,11,13,15]$ to name just a few. Related results and some background information on similar electromagnetic models are given in $[1,5,8,10,14]$. For example in [10] the authors develop theoretical results in an operator theoretic context for systems with nonlinear polarization and magnetization under global Lipschitz conditions.

We consider this paper to be a first, but nontrivial, step in analyzing the propagation of high intensity electromagnetic waves through dielectric materials using the full Maxwell's equations in the time-domain. Numerous interesting questions remain: for example, can we theoretically and/or computationally demonstrate self-focusing of the beam or wave collapse using this approach when an array of sources is used instead of a point source? These phenomena are usually discussed in the context of the nonlinear Schrödinger equation in weakly nonlinear (Kerr medium) dielectrics e.g., in $[13,15,16]$. In that approach the crucial assumption is that the relevant electric field can be represented by a finite basis of weakly coupled wavepackets and the wavepacket envelopes evolve via nonlinear equations. However, when light intensities become sufficiently high so that the material behaves like a plasma, this weak coupling theory breaks down [13]. This is exactly what happens when a laser pulse collapses into multiple self-focused filaments. The initial phases of the event are wellunderstood but as the collapse evolves an electron plasma and very large thermal gradients are generated. We hope that such phenomena can be accurately captured with our direct 
time-domain approach.

The structure of the paper is as follows: In Section 2 we present a short derivation of the model and the existence result for weak solutions. Section 3 and 4 contain the uniqueness and continuous dependence arguments, respectively. We close with a brief discussion of applications to nonlinear materials.

\section{Weak formulation and existence result}

As described in the Introduction, we consider Maxwell's equations applied to a specific physical problem as depicted in Figure 1. An infinite slab of material is interrogated by a normally incident polarized plane wave windowed pulse originating at an antenna source $z=0$ in free space $\Omega_{0}=\left[0, z_{1}\right]$. The slab of material in $\Omega=\left[z_{1}, z_{2}\right]$ is assumed to be homogeneous in the directions orthogonal to the direction $z$ of propagation of the plane wave. Under these assumptions it is possible to represent the strength of the electric and magnetic fields in $\Omega$ and $\Omega_{0}$ by the scalar functions $E(t, z)$ and $H(t, z)$, respectively. One can readily eliminate the magnetic field from the full Maxwell equations to arrive at the strong formulation of the problem (for a detailed derivation see [1])

$$
\begin{aligned}
& \mu_{0} \epsilon \ddot{E}+\mu_{0} I_{\Omega}(z) \ddot{P}+\mu_{0} \sigma \dot{E}-E^{\prime \prime}=-\mu_{0} \dot{J}_{s} \quad 0<z<z_{2}, t>0, \\
& \frac{1}{c} \frac{\partial E}{\partial t}-\left.\frac{\partial E}{\partial z}\right|_{z=0}=0 \quad t>0, \\
& E\left(t, z_{2}\right)=0 \quad t>0, \\
& E(0, z)=\Phi(z), \quad \dot{E}(0, z)=\Psi(z) \quad 0<z<z_{2},
\end{aligned}
$$

where $P$ denotes the polarization and $I_{\Omega}$ is the indicator function

$$
I_{\Omega}(z)=\left\{\begin{array}{ll}
0 & \text { if } 0<z<z_{1} \\
1 & \text { if } z_{1} \leq z \leq z_{2},
\end{array}\right\},
$$

where $z_{1}<1$ is the front boundary of the slab and $z_{2}$ is the (sometimes unknown in interrogation problems) back boundary (see Figure 1). We note that an absorbing boundary condition is placed at $z=0$ to prevent the reflection of waves. We assume that there is a supraconductive backing on the slab at $z=z_{2}$ and specify the boundary conditions accordingly. Equation (2.1) can be given in the form

$$
\frac{\epsilon}{\epsilon_{0}} \ddot{E}+\frac{1}{\epsilon_{0}} I_{\Omega}(z) \ddot{P}+\frac{1}{\epsilon_{0}} \sigma \dot{E}-c^{2} E^{\prime \prime}=-\frac{1}{\epsilon_{0}} \dot{J}_{s},
$$

where $\epsilon=\epsilon_{0}\left(1+\left(\epsilon_{r}-1\right) I_{\Omega}\right)$, and $c^{2}=\frac{1}{\epsilon_{0} \mu_{0}}$. We denote $\frac{\epsilon}{\epsilon_{0}}$ by $\hat{\varepsilon}_{r}$.

We assume that the frequency of the interrogating wave is so high that the dependence of the polarization on the electric field can no longer be adequately described by a linear constitutive law. Specifically, we consider a polarization mechanism that depends on the strength of the electric field in the convolution

$$
P(t, z)=\int_{0}^{t} g(t-s, z)(E(s, z)+f(E(s, z))) d s
$$




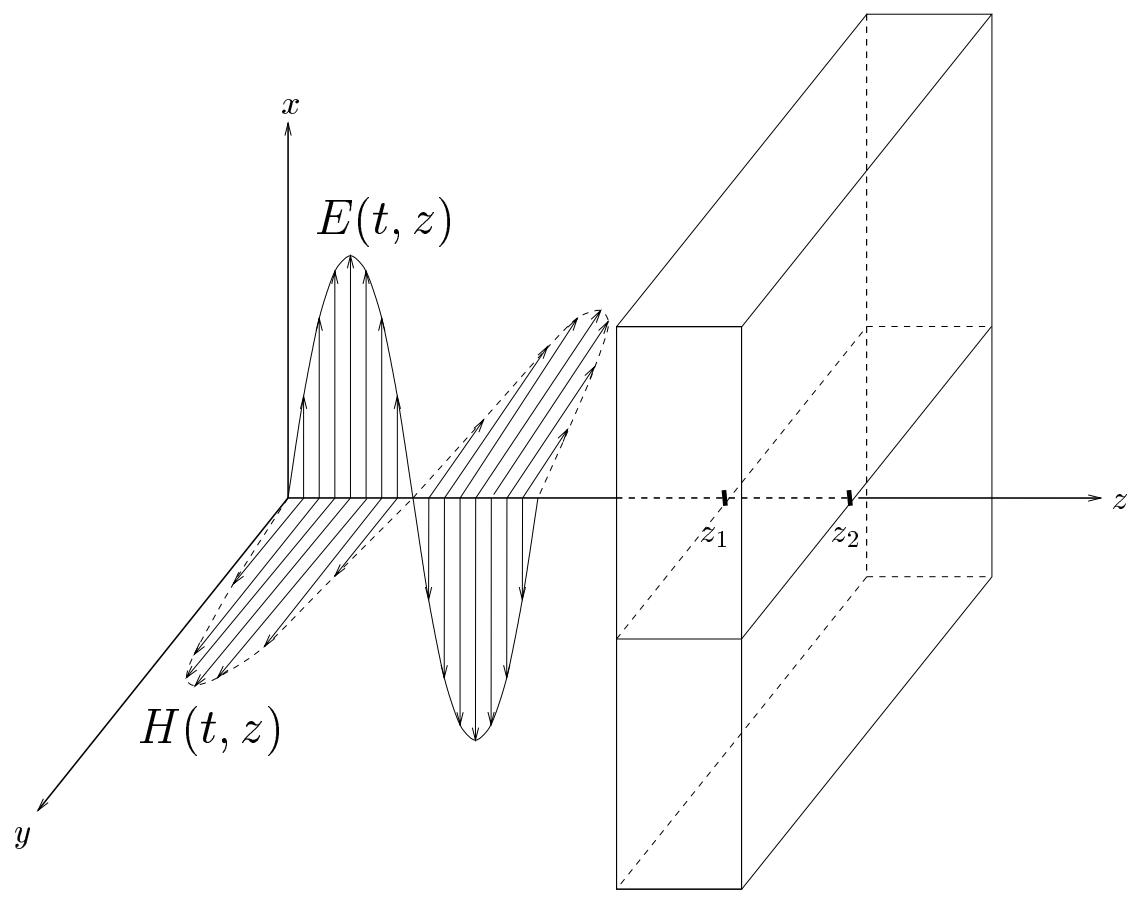

Figure 1: Geometry of the physical problem

with nonlinearity $f$. Thus

$$
\begin{aligned}
\ddot{P}(t, z) & =\int_{0}^{t} \ddot{g}(t-s, z) f(E(s, z)) d s+g(0, z) \frac{\partial}{\partial t} f(E(t, z))+\dot{g}(0, z) f(E(t, z)) \\
& +\int_{0}^{t} \ddot{g}(t-s, z) E(s, z) d s+g(0, z) \dot{E}(t, z)+\dot{g}(0, z) E(t, z),
\end{aligned}
$$

where $f: \mathbb{R} \rightarrow \mathbb{R}$ is a nonlinear (not necessarily small) perturbation of the linear mechanism. This yields the strong form of the equation

$$
\begin{aligned}
\hat{\varepsilon}_{r} \ddot{E}(t, z) & +\frac{1}{\epsilon_{0}} I_{\Omega}(z)(\sigma(z)+g(0, z)) \dot{E}(t, z) \\
& +\frac{1}{\epsilon_{0}} I_{\Omega}(z) \dot{g}(0, z) E(t, z)+\int_{0}^{t} \frac{1}{\epsilon_{0}} I_{\Omega}(z) \ddot{g}(t-s, z) E(s, z) d s \\
& +\frac{1}{\epsilon_{0}} I_{\Omega}(z) \dot{g}(0, z) f(E(t, z))+\int_{0}^{t} \frac{1}{\epsilon_{0}} I_{\Omega}(z) \ddot{g}(t-s, z) f(E(s, z)) d s \\
& +\frac{1}{\epsilon_{0}} I_{\Omega}(z) g(0, z) \frac{d}{d t} f(E(t, z))-c^{2} E^{\prime \prime}(t, z)=-\frac{1}{\epsilon_{0}} \dot{J}_{s}(t, z), \quad 0<z<z_{2} .
\end{aligned}
$$

In the physical problem $z_{2}$ is assumed to be unknown, and it is desirable to estimate it from given data. Since the existence proof is constructive in the sense that the numerical method we use to solve this problem (for both forward and inverse problems) follows the theoretical arguments, it is desirable to convert the problem to a fixed spatial domain, e.g., [0,1], as 
explained in [1]. We then do not need to update the spatial discretization and the basis functions for different $z_{2}$, but the same numerical framework can be used throughout the optimization procedure in the inverse problems (again, this is explained in detail in [1]).

We first multiply $(2.6)$ by $\phi$, where $\phi \in H^{1}\left(0, z_{2}\right)$ and integrate from 0 to $z_{2}$ using integration by parts in the last term in the left. Then we introduce a change of variable by letting

$$
\tilde{z}=h(z)=\left\{\begin{array}{ll}
z_{1} & \text { if } 0<z<z_{1} \\
z_{1}+\left(z-z_{1}\right) \frac{1-z_{1}}{z_{2}-z_{1}} & \text { if } z_{1} \leq z \leq z_{2},
\end{array}\right\}
$$

i.e., $h(z)=z+(\zeta-1)\left(z-z_{1}\right) I_{\left[z_{1}, z_{2}\right]}(z)$ with $\zeta=\frac{1-z_{1}}{z_{2}-z_{1}}$. Then $h^{\prime}(z)=1+(\zeta-1) I_{\Omega}(z)$ and $\tilde{h}^{\prime}(\tilde{z})=1+(\zeta-1) I_{\tilde{\Omega}}(\tilde{z})$, where $\tilde{\Omega}=\left[z_{1}, 1\right]$ and we adopt the notation that $\tilde{E}, \tilde{h}, \tilde{\phi}, \tilde{g}$, etc., are the maps $E, h, \phi, g$, etc., after they have been mapped from the domain $\left[0, z_{2}\right]$ to the domain $[0,1]$. Using this transformation we obtain

$$
\begin{aligned}
& \left\langle\frac{1}{\tilde{h}^{\prime}} \tilde{\hat{\varepsilon}} \frac{\tilde{E}}{\tilde{E}}(t, \cdot), \tilde{\varphi}\right\rangle+\left\langle\frac{1}{\tilde{h}^{\prime}} \frac{1}{\epsilon_{0}} I_{\tilde{\Omega}}(\tilde{\sigma}+\tilde{g}(0, \cdot)) \dot{\tilde{E}}(t, \cdot), \tilde{\varphi}\right\rangle \\
+ & \left\langle\frac{1}{\tilde{h}^{\prime}} \frac{1}{\epsilon_{0}} I_{\tilde{\Omega}} \dot{\tilde{g}}(0, \cdot) \tilde{E}(t, \cdot), \tilde{\varphi}\right\rangle+\left\langle\frac{1}{\tilde{h}^{\prime}} \int_{0}^{t} \frac{1}{\epsilon_{0}} I_{\tilde{\Omega}} \ddot{\tilde{g}}(t-s, \cdot) \tilde{E}(s, \cdot) d s, \tilde{\varphi}\right\rangle \\
+ & \left\langle\frac{1}{\tilde{h}^{\prime}} \frac{1}{\epsilon_{0}} I_{\tilde{\Omega}} \dot{\tilde{g}}(0, \cdot) f(\tilde{E}(t, \cdot)), \tilde{\varphi}\right\rangle+\left\langle\frac{1}{\tilde{h}^{\prime}} \int_{0}^{t} \frac{1}{\epsilon_{0}} I_{\tilde{\Omega}} \ddot{\tilde{g}}(t-s, \cdot) f(\tilde{E}(s, \cdot)) d s, \tilde{\varphi}\right\rangle \\
+ & \left\langle\frac{1}{\tilde{h}^{\prime}} \frac{1}{\epsilon_{0}} I_{\tilde{\Omega}} \tilde{g}(0, \cdot) \frac{d}{d t} f(\tilde{E}(t, \cdot)), \tilde{\varphi}\right\rangle+\left\langle c^{2} \tilde{h}^{\prime} \tilde{E}^{\prime}(t, \cdot), \tilde{\varphi}^{\prime}\right\rangle+c \dot{\tilde{E}}(t, 0) \tilde{\varphi}(0) \\
= & -\left\langle\frac{1}{\tilde{h}^{\prime}} \frac{1}{\epsilon_{0}} \dot{\tilde{J}}_{s}(t, \cdot), \tilde{\varphi}\right\rangle,
\end{aligned}
$$

where $\langle\cdot, \cdot\rangle$ is the $L^{2}(0,1)$ inner product. This effectively maps our system to a fixed reference domain $[0,1]$ and we use this form to define the weak solution of the problem. We let $H=L^{2}(0,1), \quad V=H_{R}^{1}(0,1)=\left\{\phi \in H^{1}(0,1) \mid \phi(1)=0\right\}$ leading to the Gelfand triple $([9,17])$ $V \hookrightarrow H \hookrightarrow V^{*}$. We say that $E \in L^{\infty}(0, T ; V)$ with $\dot{E} \in L^{2}(0, T ; H), \ddot{E} \in L^{2}\left(0, T ; V^{*}\right)$, is a weak solution if it satisfies for every $\varphi \in V$

$$
\begin{aligned}
& \left\langle\overline{\varepsilon_{r}} \ddot{E}, \varphi\right\rangle_{V^{*}, V}+\langle\gamma \dot{E}, \varphi\rangle+\langle\beta E, \varphi\rangle+\left\langle\int_{0}^{t} \alpha(t-s, \cdot) E(s, \cdot) d s, \varphi\right\rangle \\
& +\langle\beta f(E), \varphi\rangle+\left\langle\int_{0}^{t} \alpha(t-s, \cdot) f(E(s, \cdot)) d s, \varphi\right\rangle+\left\langle\hat{\gamma} \frac{d}{d t} f(E), \varphi\right\rangle \\
& +\left\langle c^{2} h^{\prime} E^{\prime}, \varphi^{\prime}\right\rangle+c \dot{E}(t, 0) \varphi(0)=\langle\mathcal{J}(t, \cdot), \varphi\rangle_{V^{*}, V}
\end{aligned}
$$

and

$$
E(0, z)=\Phi(z), \quad \dot{E}(0, z)=\Psi(z),
$$

where in (2.8) we drop the overtilda on variables and functions for simplification of notation and for $\tilde{z} \in[0,1]$, we define

$$
\alpha(t, \tilde{z})=\frac{1}{\tilde{h}^{\prime}} \frac{1}{\epsilon_{0}} I_{\tilde{\Omega}} \ddot{\tilde{g}}(t, \tilde{z}),
$$




$$
\begin{aligned}
\beta(\tilde{z}) & =\frac{1}{\tilde{h}^{\prime}} \frac{1}{\epsilon_{0}} I_{\tilde{\Omega}} \dot{\tilde{g}}(0, \tilde{z}), \\
\gamma(\tilde{z}) & =\frac{1}{\tilde{h}^{\prime}} \frac{1}{\epsilon_{0}} I_{\tilde{\Omega}}(\tilde{\sigma}(\tilde{z})+\tilde{g}(0, \tilde{z})), \\
\hat{\gamma}(\tilde{z}) & =\frac{1}{\tilde{h}^{\prime}} \frac{1}{\epsilon_{0}} I_{\tilde{\Omega}} \tilde{g}(0, \tilde{z}), \\
\mathcal{J}(t, \tilde{z}) & =-\frac{1}{\tilde{h}^{\prime}} \frac{1}{\epsilon_{0}} \tilde{\tilde{J}} s(t, \tilde{z}), \\
\overline{\varepsilon_{r}} & =\frac{1}{\tilde{h}^{\prime}} \hat{\hat{\varepsilon}}_{r} .
\end{aligned}
$$

We note that since $\frac{1}{h^{\prime}(z)}>0$ is a piecewise constant function, the inclusion of this term in $\alpha, \beta, \gamma, \hat{\gamma}, \overline{\varepsilon_{r}}, \mathcal{J}$ is essentially equivalent to using a modified $\epsilon_{0}$. In developing the theoretical existence result we can also make the simplifying assumption that $\overline{\varepsilon_{r}}=1$ without loss of generality. (This, of course, is not true for computational efforts.) Our goal is to establish the existence and uniqueness of weak solutions.

We make the following assumptions:

A1) The functions $\beta, \gamma, \hat{\gamma} \in L^{\infty}(0,1)$ with

$$
|\beta(z)| \leq L_{1}, \quad|\hat{\gamma}(z)| \leq L_{2}
$$

A2) The function $\alpha$ is bounded on $[0, T] \times(0,1)$ with

$$
|\alpha(t, z)| \leq L_{3}
$$

A3) $g(0, z) \geq 0, \sigma(z) \geq 0$. (We note that this assumption implies that $\gamma(z) \geq 0, \hat{\gamma}(z) \geq 0$ for every $z \in[0,1]$.)

A4) The nonlinear function $f: \mathbb{R} \rightarrow \mathbb{R}$ is $C^{1}$, with $f(0)=0$, and $f^{\prime}(z)>0$ for all $z \in \mathbb{R}$. $f$ is also assumed to be affine at infinity, i.e., there exist constants $R, L_{R}$ and $K_{R}$ such that for every $|x|>R,|f(x)| \leq L_{R}|x|+K_{R}$.

Remark 2.1 We note that A4) implies that $f$ is locally Lipschitz, i.e., for any $r>0$ there exists $L_{r}>0$ such that

$$
|f(x)-f(y)| \leq L_{r}|x-y| \text { for every }|x|,|y|<r \text {. }
$$

Also there is a constant $M$ with $|f(x)| \leq M|x|$ for all $x \in \mathbb{R}$.

We first prove the following theorem:

Theorem 2.1 Under assumptions A1)-A4) the system (2.8)-(2.9) has a weak solution for any $\Phi \in V, \Psi \in H$ and $\mathcal{J} \in H^{1}\left(0, T ; V^{*}\right)$. 
Proof: Our arguments are similar to those in [1] but we need additional techniques to treat the nonlinear terms. Thus we first add $\langle k E, \varphi\rangle$ to both sides of $(2.8)$ where $k$ is chosen in such a way that $\hat{\beta} \equiv k+\beta$ satisfies $\hat{\beta} \geq \varepsilon_{1}>0$ on $[0,1]$ for some constant $\varepsilon_{1}$. Such a $k$ exists since by assumption $\beta \in L^{\infty}(0,1)$. Next we define the sesquilinear form $\sigma_{1}: V \times V \rightarrow \mathbb{C}$ by

$$
\sigma_{1}(\phi, \psi)=\left\langle c^{2} h^{\prime} \phi^{\prime}, \psi^{\prime}\right\rangle_{H}+\langle\hat{\beta} \phi, \psi\rangle_{H} \quad \text { for } \phi, \psi \in V .
$$

It is readily seen that $\sigma_{1}$ is $\mathrm{V}$-continuous and V-elliptic, i.e., there are positive constants $c_{1}, c_{2}$ such that

$$
\begin{aligned}
\left|\sigma_{1}(\phi, \psi)\right| & \leq c_{2}\|\phi\|_{V}\|\psi\|_{V} \text { for all } \phi, \psi \in V \\
\sigma_{1}(\phi, \phi) & \geq c_{1}\|\phi\|_{V}^{2} \text { for all } \phi \in V .
\end{aligned}
$$

Let $\Phi \in V$ and $\Psi \in H$ and fix $T>0$. We choose a subset $\left\{w_{i}\right\}_{i=1}^{\infty}$ spanning V. Without loss of generality we assume that the elements $w_{i}$ are linearly independent. Let $V^{m}=$ $\operatorname{span}\left\{w_{1}, \ldots, w_{m}\right\}$, and define the Galerkin approximations

$$
E_{m}(t, z)=\sum_{i=1}^{m} e_{i}^{m}(t) w_{i}(z)
$$

where the $\left\{e_{i}^{m}(t)\right\}_{i=1}^{m}$ are obtained by solving the m-dimensional system of nonlinear delay differential equations:

$$
\begin{aligned}
& \left\langle\ddot{E}_{m}(t), w_{i}\right\rangle_{H}+\left\langle\gamma \dot{E}_{m}(t), w_{i}\right\rangle_{H}+\left\langle\int_{0}^{t} \alpha(t-s, \cdot) E_{m}(s, \cdot) d s, w_{i}\right\rangle \\
& +\sigma_{1}\left(E_{m}(t), w_{i}\right)+c \dot{E}_{m}(t, 0) w_{i}(0)+\left\langle\hat{\gamma} \frac{d}{d t} f\left(E_{m}(t)\right), w_{i}\right\rangle+\left\langle\int_{0}^{t} \alpha(t-s, \cdot) f\left(E_{m}(s, \cdot)\right) d s, w_{i}\right\rangle \\
& +\left\langle\beta f\left(E_{m}(t)\right), w_{i}\right\rangle=\left\langle\mathcal{J}(t), w_{i}\right\rangle_{V^{*}, V}+\left\langle k E_{m}(t), w_{i}\right\rangle,
\end{aligned}
$$

for $i=1 \ldots m$, with

$$
\begin{aligned}
& E_{m}(0)=\Phi^{m} \\
& \dot{E}_{m}(0)=\Psi^{m},
\end{aligned}
$$

where $\Phi^{m} \rightarrow \Phi$ in $V$, and $\Psi^{m} \rightarrow \Psi$ in H. Multiplying each equation by $\dot{e}_{i}^{m}(t)$ and adding them we obtain

$$
\begin{aligned}
& \frac{1}{2} \frac{d}{d t}\left\|\dot{E}_{m}(t)\right\|_{H}^{2}+\left\langle\gamma \dot{E}_{m}(t), \dot{E}_{m}(t)\right\rangle_{H}+\left\langle\int_{0}^{t} \alpha(t-s, \cdot) E_{m}(s, \cdot) d s, \dot{E}_{m}(t)\right\rangle_{H} \\
& +\sigma_{1}\left(E_{m}(t), \dot{E}_{m}(t)\right)+c\left|\dot{E}_{m}(t, 0)\right|^{2}+\left\langle\hat{\gamma} \frac{d}{d t} f\left(E^{m}(t)\right), \dot{E}_{m}(t)\right\rangle_{H} \\
& +\left\langle\int_{0}^{t} \alpha(t-s, \cdot) f\left(E_{m}(s, \cdot)\right) d s, \dot{E}_{m}(t)\right\rangle_{H}+\left\langle\beta f\left(E_{m}(t)\right), \dot{E}_{m}(t)\right\rangle_{H} \\
& =\left\langle\mathcal{J}(t), \dot{E}_{m}(t)\right\rangle_{V^{*}, V}+\left\langle k E_{m}(t), \dot{E}_{m}(t)\right\rangle_{H} \\
& E_{m}(0)=\Phi_{m} \\
& \dot{E}_{m}(0)=\Psi_{m}
\end{aligned}
$$


Thus,

$$
\begin{aligned}
& \frac{1}{2} \frac{d}{d t}\left\{\left\|\dot{E}_{m}(t)\right\|_{H}^{2}+\sigma_{1}\left(E_{m}(t), E_{m}(t)\right)\right\}+\left\|\sqrt{\gamma+\hat{\gamma} f^{\prime}\left(E_{m}(t)\right)} \dot{E}_{m}(t)\right\|_{H}^{2}+c\left|\dot{E}_{m}(t, 0)\right|^{2} \\
& =\left\langle-\int_{0}^{t} \alpha(t-s, \cdot) E_{m}(s, \cdot) d s, \dot{E}_{m}(t)\right\rangle_{H}+\left\langle-\int_{0}^{t} \alpha(t-s, \cdot) f\left(E_{m}(s, \cdot)\right) d s, \dot{E}_{m}(t)\right\rangle_{H} \\
& +\left\langle-\beta f\left(E_{m}(t)\right), \dot{E}_{m}(t)\right\rangle_{H}+\left\langle\mathcal{J}(t), \dot{E}_{m}(t)\right\rangle_{V^{*}, V}+\left\langle k E_{m}(t), \dot{E}_{m}(t)\right\rangle_{H},
\end{aligned}
$$

where we used that $\gamma+\hat{\gamma} f^{\prime}(E) \geq 0$ by assumptions A3) and A4). Integration from 0 to t, where $t \in[0, T]$ yields

$$
\begin{aligned}
& \left\|\dot{E}_{m}(t)\right\|_{H}^{2}+c_{1}\left\|E_{m}(t)\right\|_{V}^{2}+2 \int_{0}^{t}\left\|\sqrt{\gamma+\hat{\gamma} f^{\prime}\left(E_{m}(s)\right)} \dot{E}_{m}(s)\right\|_{H}^{2} d s+2 c\left|\dot{E}_{m}(\cdot, 0)\right|_{L^{2}(0, t)} \\
& \leq\left\|\dot{E}_{m}(0)\right\|_{H}^{2}+c_{2}\left\|E_{m}(0)\right\|_{V}^{2}+2\left|\int_{0}^{t} F_{m}(\tau) d \tau\right|
\end{aligned}
$$

with

$$
\begin{aligned}
F_{m}(\tau) & =\left\langle-\int_{0}^{\tau} \alpha(\tau-s, \cdot) E_{m}(s, \cdot) d s, \dot{E}_{m}(\tau)\right\rangle_{H}+\left\langle k E_{m}(\tau), \dot{E}_{m}(\tau)\right\rangle_{H} \\
& +\left\langle\mathcal{J}(\tau), \dot{E}_{m}(\tau)\right\rangle_{V^{*}, V}+\left\langle-\int_{0}^{\tau} \alpha(\tau-s, \cdot) f\left(E_{m}(s, \cdot)\right) d s, \dot{E}_{m}(\tau)\right\rangle_{H} \\
& +\left\langle-\beta f\left(E_{m}(\tau)\right), \dot{E}_{m}(\tau)\right\rangle_{H} \\
& \equiv T_{1}(\tau)+T_{2}(\tau)+T_{3}(\tau)+T_{4}(\tau)+T_{5}(\tau) .
\end{aligned}
$$

Let $0<\tau<t$ be arbitrary. We next estimate the terms $T_{i}(\tau)$ one by one. Thus,

$$
\begin{aligned}
\left|T_{1}(\tau)\right| & \leq \int_{0}^{\tau} L_{3}\left\|E_{m}(s)\right\|_{H} d s\left\|\dot{E}_{m}(\tau)\right\|_{H} \\
& \leq \frac{1}{2}\left(\int_{0}^{\tau} L_{3}\left\|E_{m}(s)\right\|_{H} d s\right)^{2}+\frac{1}{2}\left\|\dot{E}_{m}(\tau)\right\|_{H}^{2} \\
& \leq \frac{1}{2} L_{3}^{2} t \int_{0}^{t}\left\|E_{m}(s)\right\|_{H}^{2} d s+\frac{1}{2}\left\|\dot{E}_{m}(\tau)\right\|^{2} .
\end{aligned}
$$

Using this estimate we obtain

$$
\int_{0}^{t}\left|T_{1}(\tau)\right| d \tau \leq \frac{1}{2} L_{3}^{2} t^{2} \int_{0}^{t}\left\|E_{m}(s)\right\|_{H}^{2} d s+\frac{1}{2} \int_{0}^{t}\left\|\dot{E}_{m}(s)\right\|_{H}^{2} d s .
$$

Next, we have

$$
\begin{aligned}
\int_{0}^{t}\left|T_{2}(\tau)\right| d \tau & \leq \int_{0}^{t}\left|\left\langle k E_{m}(\tau), \dot{E}_{m}(\tau)\right\rangle\right| d \tau \\
& \leq \int_{0}^{t}\left\{\frac{1}{2} k^{2}\left\|E_{m}(\tau)\right\|_{H}^{2}+\frac{1}{2}\left\|\dot{E}_{m}(\tau)\right\|_{H}^{2}\right\} d \tau
\end{aligned}
$$

Since $\dot{\mathcal{J}} \in L^{2}\left(0, T ; V^{*}\right)$ we have

$$
\frac{d}{d t}\left\langle\mathcal{J}(t), E_{m}(t)\right\rangle_{V^{*}, V}=\left\langle\dot{\mathcal{J}}(t), E_{m}(t)\right\rangle_{V^{*}, V}+\left\langle\mathcal{J}(t), \dot{E}_{m}(t)\right\rangle_{V^{*}, V}
$$


Using this relationship we have the estimate for the third term:

$$
\begin{aligned}
\left|\int_{0}^{t} T_{3}(\tau) d \tau\right| & \leq\left|\int_{0}^{t}\left\{\frac{d}{d \tau}\left\langle\mathcal{J}(\tau), E_{m}(\tau)\right\rangle_{V^{*}, V}-\left\langle\dot{\mathcal{J}}(\tau), E_{m}(\tau)\right\rangle_{V^{*}, V}\right\} d \tau\right| \\
& =\left|\left\langle\mathcal{J}(t), E_{m}(t)\right\rangle_{V^{*}, V}-\left\langle\mathcal{J}(0), E_{m}(0)\right\rangle_{V^{*}, V}-\int_{0}^{t}\left\langle\dot{\mathcal{J}}(\tau), E_{m}(\tau)\right\rangle_{V^{*}, V} d \tau\right| \\
& \leq \frac{1}{2 \delta}\|\mathcal{J}(t)\|_{V^{*}}^{2}+\delta\left\|E_{m}(t)\right\|_{V}^{2}+\frac{1}{2}\|\mathcal{J}(0)\|_{V^{*}}^{2}+\frac{1}{2}\left\|E_{m}(0)\right\|_{V}^{2} \\
& +\int_{0}^{t}\left\{\frac{1}{2}\|\dot{\mathcal{J}}(\tau)\|_{V^{*}}^{2}+\frac{1}{2}\left\|E_{m}(\tau)\right\|_{V}^{2}\right\} d \tau
\end{aligned}
$$

where $\delta>0$ is arbitrary. Using the assumptions A2) and A4) we can estimate the next term as above to yield

$$
\begin{aligned}
\left|T_{4}(\tau)\right| & =\left|\left\langle\int_{0}^{\tau} \alpha(\tau-s, \cdot) f\left(E_{m}(s, \cdot)\right) d s, \dot{E}_{m}(\tau)\right\rangle_{H}\right| \\
& \leq \int_{0}^{\tau} L_{3}\left\|f\left(E_{m}(s)\right)\right\|_{H} d s\left\|\dot{E}_{m}(\tau)\right\| \\
& \leq \frac{1}{2} L_{3}^{2} M^{2} t \int_{0}^{t}\left\|E_{m}(s)\right\|_{H}^{2} d s+\frac{1}{2}\left\|\dot{E}_{m}(\tau)\right\|_{H}^{2} .
\end{aligned}
$$

Thus we find

$$
\int_{0}^{t}\left|T_{4}(\tau)\right| d \tau \leq \frac{1}{2} L_{3}^{2} M^{2} t^{2} \int_{0}^{t}\left\|E_{m}(\tau)\right\|_{H}^{2} d \tau+\frac{1}{2} \int_{0}^{t}\left\|\dot{E}_{m}(\tau)\right\|_{H}^{2} d \tau .
$$

Similarly the last term can be estimated

$$
\begin{aligned}
\int_{0}^{t}\left|T_{5}(\tau)\right| d \tau & \leq \int_{0}^{t}\left|\left\langle\beta f\left(E_{m}(\tau)\right), \dot{E}_{m}(\tau)\right\rangle\right| d \tau \\
& \leq \int_{0}^{t} L_{1}\left\|f\left(E_{m}(\tau)\right)\right\|_{H}\left\|\dot{E}_{m}(\tau)\right\|_{H} d \tau \\
& \leq \int_{0}^{t} \frac{1}{2} L_{1}^{2} M^{2}\left\|E_{m}(\tau)\right\|_{H}^{2}+\frac{1}{2}\left\|\dot{E}_{m}(\tau)\right\|_{H}^{2} d \tau .
\end{aligned}
$$

Combining the estimates (2.16)-(2.20) we obtain from (2.15)

$$
\begin{aligned}
& \left\|\dot{E}_{m}(t)\right\|_{H}^{2}+\left(c_{1}-2 \delta\right)\left\|E_{m}(t)\right\|_{V}^{2}+2 c\left\|\dot{E}_{m}(\cdot, 0)\right\|_{L^{2}(0, t)}^{2} \\
& \leq\left\|\dot{E}_{m}(0)\right\|_{H}^{2}+\left(c_{2}+1\right)\left\|E_{m}(0)\right\|_{V}^{2}+\|\mathcal{J}(0)\|_{V^{*}}^{2}+\int_{0}^{t}\|\dot{\mathcal{J}}(\tau)\|_{V^{*}}^{2} d \tau \\
& +\left(L_{3}^{2} t^{2}+k^{2}+L_{3}^{2} M^{2} t^{2}+L_{1}^{2} M^{2}\right) \int_{0}^{t}\left\|E_{m}(\tau)\right\|_{H}^{2} d \tau+\int_{0}^{t}\left\|E_{m}(\tau)\right\|_{V}^{2} d \tau+\frac{2}{\delta}\|\mathcal{J}(t)\|_{V^{*}}^{2} \\
& +4 \int_{0}^{t}\left\|\dot{E}_{m}(\tau)\right\|_{H}^{2} d \tau .
\end{aligned}
$$

We choose $\delta$ to be such that $\nu=c_{1}-2 \delta>0$. Since $V \hookrightarrow H$ there exists a constant $\mu>0$ with $\|u\|_{H} \leq \mu\|u\|_{V}$, for every $u \in V$. Also, $E_{m}(0)$ is bounded in $V, \dot{E}_{m}(0)$ is bounded in $H$ 
and $\mathcal{J} \in H^{1}\left(0, T ; V^{*}\right)$, so we can conclude that

$$
\begin{aligned}
& \left\|\dot{E}_{m}(t)\right\|_{H}^{2}+\nu\left\|E_{m}(t)\right\|_{V}^{2}+2 c\left\|\dot{E}_{m}(\cdot, 0)\right\|_{L^{2}(0, t)}^{2} \leq B_{1}+4 \int_{0}^{t}\left\|\dot{E}_{m}(\tau)\right\|_{H}^{2} d \tau \\
& +B_{2} \int_{0}^{t}\left\|E_{m}(\tau)\right\|_{V}^{2} d \tau, \text { for all } t \in[0, T]
\end{aligned}
$$

where $B_{1}, B_{2}$ are independent of $\mathrm{m}$. Now by the Gronwall inequality we arrive at

$$
\left\|\dot{E}_{m}(t)\right\|_{H}^{2}+\nu\left\|E_{m}(t)\right\|_{V}^{2}+2 c\left\|\dot{E}_{m}(\cdot, 0)\right\|_{L^{2}(0, t)}^{2} \leq C(T, f, g, \Phi, \Psi, \mathcal{J})
$$

where $C$ is a positive constant independent of $m$. Hence

$$
\begin{aligned}
& \left\{E_{m}\right\} \text { is bounded in } C(0, T ; V) \subset L^{2}(0, T ; V) \\
& \left\{\dot{E}_{m}\right\} \text { is bounded in } C(0, T ; H) \subset L^{2}(0, T ; H) \text { and } \\
& \left\{\dot{E}_{m}(\cdot, 0)\right\} \text { is bounded in } L^{2}(0, T) .
\end{aligned}
$$

Consequently there exist subsequences (again denoted by the subscript m) such that

$$
\begin{aligned}
E_{m} & \rightarrow E \text { weakly in } L^{2}(0, T ; V) \\
\dot{E}_{m} & \rightarrow \tilde{E} \text { weakly in } L^{2}(0, T ; H) \\
\dot{E}_{m}(\cdot, 0) & \rightarrow E_{L} \text { weakly in } L^{2}(0, T) .
\end{aligned}
$$

First we show that $\tilde{E}=\dot{E}, E_{L}=\dot{E}(\cdot, 0)$ in some sense. For each $m$ we have that

$$
\begin{aligned}
E_{m}(t) & =E_{m}(0)+\int_{0}^{t} \dot{E}_{m}(s) d s \\
E_{m}(t, 0) & =E_{m}(0,0)+\int_{0}^{t} \dot{E}_{m}(s, 0) d s .
\end{aligned}
$$

Passing to the limit in (2.29) (in the weak $H$ sense) and in (2.30), we obtain

$$
\begin{aligned}
E(t) & =\Phi+\int_{0}^{t} \tilde{E}(s) d s \\
E(t, 0) & =\Phi(0)+\int_{0}^{t} E_{L}(s, 0) d s
\end{aligned}
$$

where (2.31) holds in the sense of $H$ for each $t \in[0, T]$. Thus (2.31) implies that $\tilde{E}=\dot{E}$, while from (2.32) we can conclude that $E(t, 0)$ exists and is continuous in $t$. Actually, $E(t, 0)$ is absolutely continuous with $\dot{E}(t, 0)=E_{L}(t)$ for almost every t.

We proceed to show that the convergence (2.26) can be strengthened to provide the strong convergence $E_{m} \rightarrow E$ in $C(0, T ; H)$. We note that this strong convergence enables us to pass to the limit as $m \rightarrow \infty$ in expressions involving the nonlinear function of $E_{m}$, and thus it is the key to establishing the existence of weak solutions for systems with nonlinear polarization with the techniques employed here. To achieve this result we use the following version of the Arzela-Ascoli theorem (see [12], p.149): 
Arzela-Ascoli theorem: If $Y$ is a complete metric space and $\mathcal{F} \subset C(0, T ; Y)$, then $\mathcal{F}$ is relatively compact if and only if $\mathcal{F}$ is equicontinuous and $\{f(t): f \in \mathcal{F}\}$ is relatively compact in $Y$ for each $t \in[0, T]$.

Here we let $Y=H$ and $\mathcal{F}=\left\{E_{m}\right\}_{m=1}^{\infty} \subset C(0, T ; H)$. First we show that the subset $\left\{E_{m}\right\}$ is equicontinuous in $C(0, T ; H)$. Using the estimate $(2.21)$ we have

$$
\begin{aligned}
\left\|E_{m}(t+\Delta t)-E_{m}(t)\right\|_{H}^{2} & =\left\|\int_{t}^{t+\Delta t} \dot{E}_{m}(\tau) d \tau\right\|_{H}^{2} \\
& \leq\left(\int_{t}^{t+\Delta t}\left\|\dot{E}_{m}(\tau)\right\|_{H} d \tau\right)^{2} \\
& \leq \Delta t \int_{0}^{t+\Delta t}\left\|\dot{E}_{m}(\tau)\right\|_{H}^{2} d \tau \\
& \leq(\Delta t)^{2} C
\end{aligned}
$$

Since for each $t \in[0, T]\left\{E_{m}(t)\right\}_{m=1}^{\infty}$ is uniformly bounded in $V$ by the a priori estimate (2.22) and $V$ is compactly embedded in $H$ we can conclude that $\left\{E_{m}(t)\right\}_{m=1}^{\infty}$ is relatively compact in $H$ for each $t \in[0, T]$. Thus the Arzela-Ascoli theorem guarantees that $\left\{E_{m}(t)\right\}_{m=1}^{\infty}$ is relatively compact in $C(0, T ; H)$ and hence there exists a subsequence (again denoted by $\left.E_{m}\right)$ that converges strongly to $E$ in $C(0, T ; H)$. Now we claim that $E$ (which must be equivalent to the limit in (2.26)) is a weak solution of (2.8)-(2.9). To establish this fact we first take $\psi \in C^{1}(0, T)$ with $\psi(T)=0$ and choose $\psi_{j}(t) \equiv \psi(t) w_{j}$ where $\left\{w_{j}\right\}_{j=1}^{\infty}$ are the basis elements as before. For a fixed $j$ we have that $E_{m}$ satisfies the following relation for all $m>j$ :

$$
\begin{aligned}
& \int_{0}^{T}\left\{\left\langle\ddot{E}_{m}(t), \psi_{j}(t)\right\rangle+\left\langle\left(\gamma+\hat{\gamma} f^{\prime}\left(E_{m}(t)\right)\right) \dot{E}_{m}(t), \psi_{j}(t)\right\rangle+\sigma_{1}\left(E_{m}(t), \psi_{j}(t)\right)\right. \\
& +\left\langle\int_{0}^{t} \alpha(t-s, \cdot) E_{m}(s, \cdot) d s, \psi_{j}(t)\right\rangle+c \dot{E}_{m}(t, 0) \psi_{j}(t, 0)+\left\langle\int_{0}^{t} \alpha(t-s, \cdot) f\left(E_{m}(s, \cdot)\right) d s, \psi_{j}(t)\right\rangle \\
& \left.+\left\langle\beta f\left(E_{m}(t)\right), \psi_{j}(t)\right\rangle\right\} d t=\int_{0}^{T}\left\{\left\langle\mathcal{J}(t), \psi_{j}(t)\right\rangle_{V, V^{*}}+\left\langle k E_{m}(t), \psi_{j}(t)\right\rangle\right\} d t
\end{aligned}
$$

We integrate by parts in the first term in the left using the property that $\psi(T)=0$ to obtain

$$
\begin{aligned}
& \int_{0}^{T}\left\{-\left\langle\dot{E}_{m}(t), \dot{\psi}_{j}(t)\right\rangle+\left\langle\left(\gamma+\hat{\gamma} f^{\prime}\left(E_{m}(t)\right)\right) \dot{E}_{m}(t), \psi_{j}(t)\right\rangle+\sigma_{1}\left(E_{m}(t), \psi_{j}(t)\right)\right. \\
& +\left\langle\int_{0}^{t} \alpha(t-s, \cdot) E_{m}(s, \cdot) d s, \psi_{j}(t)\right\rangle+c \dot{E}_{m}(t, 0) \psi_{j}(t, 0)+\left\langle\int_{0}^{t} \alpha(t-s, \cdot) f\left(E_{m}(s, \cdot)\right) d s, \psi_{j}(t)\right\rangle \\
& \left.+\left\langle\beta f\left(E_{m}(t)\right), \psi_{j}(t)\right\rangle\right\} d t=\int_{0}^{T}\left\{\left\langle\mathcal{J}(t), \psi_{j}(t)\right\rangle_{V^{*}, V}+\left\langle k E_{m}(t), \psi_{j}(t)\right\rangle\right\} d t+\left\langle\Psi, \psi_{j}(0)\right\rangle .
\end{aligned}
$$

Now we can take the limit as $m \rightarrow \infty$ using the convergences (2.26)-(2.28) and the strong convergence of $E_{m} \rightarrow E$ in $C(0, T ; H)$ to obtain

$$
\int_{0}^{T}\left\{-\left\langle\dot{E}(t), \dot{\psi}_{j}(t)\right\rangle+\left\langle\left(\gamma+\hat{\gamma} f^{\prime}(E(t))\right) \dot{E}(t), \psi_{j}(t)\right\rangle+\sigma_{1}\left(E(t), \psi_{j}(t)\right)\right.
$$




$$
\begin{aligned}
& +\left\langle\int_{0}^{t} \alpha(t-s, \cdot) E(s, \cdot) d s, \psi_{j}(t)\right\rangle+c \dot{E}(t, 0) \psi_{j}(t, 0)+\left\langle\int_{0}^{t} \alpha(t-s, \cdot) f(E(s, \cdot)) d s, \psi_{j}(t)\right\rangle \\
& \left.+\left\langle\beta f(E(t)), \psi_{j}(t)\right\rangle\right\} d t=\int_{0}^{T}\left\{\left\langle\mathcal{J}(t), \psi_{j}(t)\right\rangle_{V^{*}, V}+\left\langle k E(t), \psi_{j}(t)\right\rangle\right\} d t+\left\langle\Psi, \psi_{j}(0)\right\rangle .
\end{aligned}
$$

We note that passing to the limit in the second term under the integral in the left is possible by the convergences (2.26), the strong convergence $E_{m} \rightarrow E$ in $C(0, T ; H)$ and the fact that $f^{\prime}$ is assumed to be continuous. It follows that for every $\psi_{j}$ we have in the $L^{2}(0, T)$ sense (except in the first term which is in the distributional sense in $t$ )

$$
\begin{aligned}
& \left.\frac{d}{d t}\left\langle\dot{E}(t), \psi_{j}(t)\right\rangle+\left\langle\gamma+\hat{\gamma} f^{\prime}(E(t))\right) \dot{E}(t), \psi_{j}(t)\right\rangle+\sigma_{1}\left(E(t), \psi_{j}(t)\right) \\
& +\left\langle\int_{0}^{t} \alpha(t-s, \cdot) E(s) d s, \psi_{j}(t)\right\rangle+c \dot{E}(t, 0) \psi_{j}(t, 0)+\left\langle\int_{0}^{t} \alpha(t-s, \cdot) f(E(s)) d s, \psi_{j}(t)\right\rangle \\
& +\left\langle\beta f(E(t)), \psi_{j}(t)\right\rangle=\left\langle\mathcal{J}(t), \psi_{j}(t)\right\rangle_{V^{*}, V}+\left\langle k E(t), \psi_{j}(t)\right\rangle .
\end{aligned}
$$

Since $\left\{w_{j}\right\}_{j=1}^{\infty}$ is total in $V$ we can conclude that $\ddot{E} \in L^{2}\left(0, T ; V^{*}\right)$ and $E$ satisfies (2.8). From (2.31) it follows that $E(0)=\Phi$ and the fact that $\dot{E}(0)=\Psi$ can be established exactly as in [4]. Hence we find that $E$ is a solution of (2.8)-(2.9). It remains to argue that $E \in L^{\infty}(0, T ; V)$. We show using the Arzela-Ascoli theorem that the Galerkin approximations satisfy an additional convergence property, namely, that $E_{m} \rightarrow E$ in $C_{W}(0, T ; V)$. Since this implies $E_{m}(t) \rightarrow E(t)$ weakly in $\mathrm{V}$, we can conclude from the a priori estimate (2.22) that for all $t \in[0, T]$

$$
\|E(t)\|_{V}^{2} \leq \frac{1}{\nu} C
$$

and hence $E \in L^{\infty}(0, T ; V)$.

To establish the equicontinuity of $\left\{E_{m}\right\}$ in $C_{W}(0, T ; V)$ we use similar arguments as those in [2]. Namely, we note that the mapping $A_{1}: V \rightarrow V^{*}:\left\langle A_{1} \phi, \psi\right\rangle_{V^{*}, V}=\sigma_{1}(\phi, \psi)$ is a topological isomorphism. We define $\operatorname{dom} A_{1}=\left\{v \in V: A_{1} v \in H\right\}=A_{1}^{-1}(H)$. Since $\mathrm{H}$ is dense in $V^{*}, \operatorname{dom} A_{1}$ is dense in $\mathrm{V}$. We assume that $V$ is equipped with the inner product $\sigma_{1}(\cdot, \cdot)$, which is in fact equivalent to the original inner product in $\mathrm{V}$. Let us first take $v \in \operatorname{dom} A_{1}$. Then we have

$$
\begin{aligned}
& \left|\sigma_{1}\left(v, E_{m}(t+\Delta t)-E_{m}(t)\right)\right|=\left|\left\langle A_{1} v, E_{m}(t+\Delta t)-E_{m}(t)\right\rangle\right| \leq\left\|A_{1} v\right\| \int_{t}^{t+\Delta t}\left\|\dot{E}_{m}(\tau)\right\| d \tau \\
& \leq\left\|A_{1} v\right\| \sqrt{C}|\Delta t|,
\end{aligned}
$$

where $C$ is the constant from (2.22). Now let $\phi \in V$ be arbitrary, $v \in \operatorname{dom} A_{1}$, and $\epsilon>0$.

$$
\begin{aligned}
& \left|\sigma_{1}\left(\phi, E_{m}(t+\Delta t)-E_{m}(t)\right)\right| \leq\left|\sigma_{1}\left(v, E_{m}(t+\Delta t)-E_{m}(t)\right)\right| \\
& +\left|\sigma_{1}\left(\phi-v, E_{m}(t+\Delta t)-E_{m}(t)\right)\right| \leq\left\|A_{1} v\right\| \sqrt{C}|\Delta t|+2 C\|\phi-v\|_{V} .
\end{aligned}
$$

Since $\operatorname{dom} A_{1}$ is dense in $\mathrm{V}$, we can choose $v \in \operatorname{dom} A_{1}$ such that $2 C\|\phi-v\|_{V}<\frac{\epsilon}{2}$. Thus if $|\Delta t|<\delta=\frac{\epsilon}{2\left\|A_{1} v\right\| \sqrt{C}}$, then

$$
\left|\sigma_{1}\left(\phi, E_{m}(t+\Delta t)-E_{m}(t)\right)\right| \leq \epsilon
$$


which establishes the desired equicontinuity. Since $\left\{E_{m}(t)\right\}$ is bounded in V, uniformly in $\mathrm{t}$, we can choose $\mathrm{Y}$ in the Arzela-Ascoli theorem to be a closed and bounded subset of $\mathrm{V}$ containing $\left\{E_{m}(t)\right\}$. We equip $\mathrm{Y}$ with the weak topology. Thus $\mathrm{Y}$ is a compact metric space, and $\left\{E_{m}(t)\right\}$ is relatively compact in $\mathrm{Y}$ for each t. Since we already established the equicontinuity of $\left\{E_{m}\right\}$ in $C_{W}(0, T ; V)$, we obtain by the Arzela-Ascoli theorem that $\left\{E_{m}\right\}$ is relatively compact in $C(0, T ; Y)$, i.e., we have $E_{m}(t) \rightarrow E(t)$ weakly in $\mathrm{V}$ uniformly in $t$ for a suitable subsequence. Thus $E \in L^{\infty}(0, T ; V)$ and Theorem 2.1. is proved.

\section{Uniqueness}

We next establish the uniqueness of weak solutions in $L^{\infty}(0, T ; V) \cap C(0, T ; H)$. Thus suppose that $E_{1}, E_{2}$ both solve (2.8)-(2.9) with the same data $\Phi, \Psi, \mathcal{J}$. Then $E \equiv E_{1}-E_{2}$ satisfies $E(0, z)=0, \dot{E}(0, z)=0$ and

$$
\begin{aligned}
& \langle\ddot{E}, \varphi\rangle_{V^{*}, V}+\langle\gamma \dot{E}, \varphi\rangle+\sigma_{1}(E, \varphi)+\left\langle\int_{0}^{t} \alpha(t-s, \cdot) E(s, \cdot) d s, \varphi\right\rangle+\left\langle\beta\left(f\left(E_{1}\right)-f\left(E_{2}\right)\right), \varphi\right\rangle \\
& +\left\langle\int_{0}^{t} \alpha(t-s, \cdot)\left(f\left(E_{1}(s, \cdot)\right)-f\left(E_{2}(s, \cdot)\right)\right) d s, \varphi\right\rangle+\left\langle\hat{\gamma} \frac{d}{d t}\left(f\left(E_{1}\right)-f\left(E_{2}\right)\right), \varphi\right\rangle \\
& +c \dot{E}(t, 0) \varphi(0)-k\langle E, \varphi\rangle=0 \text { for every } \varphi \in V .
\end{aligned}
$$

For arbitrary but fixed $\nu$ in $(0, T)$ define

$$
\Psi_{\nu}(t)= \begin{cases}-\int_{t}^{\nu} E(\xi) d \xi & \text { for } t<\nu, \\ 0 & \text { for } t \geq \nu\end{cases}
$$

so $\Psi_{\nu}(T)=0$ and $\Psi_{\nu}(t) \in V$ for each $t \in[0, T]$. We also have that

$$
\int_{0}^{\nu}\left\langle\ddot{E}(t), \Psi_{\nu}(t)\right\rangle_{V^{*}, V}+\langle\dot{E}(t), E(t)\rangle d t=\int_{0}^{\nu} \frac{d}{d t}\left\langle\dot{E}(t), \Psi_{\nu}(t)\right\rangle d t=0 .
$$

Let $\varphi=\Psi_{\nu}(t)$ in (3.37) and integrate over t from 0 to $\nu$ :

$$
\begin{aligned}
& \int_{0}^{\nu}\left\{\langle\dot{E}(t), E(t)\rangle-\left\langle\gamma \dot{E}(t), \Psi_{\nu}(t)\right\rangle-\sigma_{1}\left(E(t), \Psi_{\nu}(t)\right)-\left\langle\int_{0}^{t} \alpha(t-s, \cdot) E(s, \cdot) d s, \Psi_{\nu}(t)\right\rangle\right. \\
& -\left\langle\beta\left(f\left(E_{1}(t)\right)-f\left(E_{2}(t)\right)\right), \Psi_{\nu}(t)\right\rangle-\left\langle\int_{0}^{t} \alpha(t-s, \cdot)\left(f\left(E_{1}(s, \cdot)\right)-f\left(E_{2}(s, \cdot)\right)\right) d s, \Psi_{\nu}(t)\right\rangle \\
& \left.-\left\langle\hat{\gamma} \frac{d}{d t}\left(f\left(E_{1}(t)\right)-f\left(E_{2}(t)\right)\right), \Psi_{\nu}(t)\right\rangle-c \dot{E}(t, 0) \Psi_{\nu}(t)(0)+k\left\langle E(t), \Psi_{\nu}(t)\right\rangle\right\} d t=0 .(3.39)
\end{aligned}
$$

By using the relations

$$
\begin{aligned}
& \int_{0}^{\nu}\left\langle\gamma \dot{E}, \Psi_{\nu}\right\rangle+\langle\gamma E, E\rangle d t=\int_{0}^{\nu} \frac{d}{d t}\left\langle\gamma E, \Psi_{\nu}\right\rangle d t=0 \\
& \frac{d}{d t} \sigma_{1}\left(\Psi_{\nu}(t), \Psi_{\nu}(t)\right)=2 \sigma_{1}\left(E(t), \Psi_{\nu}(t)\right)
\end{aligned}
$$




$$
\begin{aligned}
& \int_{0}^{\nu}\left\{c \dot{E}(t, 0) \Psi_{\nu}(t)(0)+c E(t, 0)^{2}\right\} d t=\int_{0}^{\nu} \frac{d}{d t} c E(t, 0) \Psi_{\nu}(t)(0) d t=0 \\
& \frac{d}{d t}\left\langle k \Psi_{\nu}(t), \Psi_{\nu}(t)\right\rangle=2\left\langle k E(t), \Psi_{\nu}(t)\right\rangle \\
& \int_{0}^{\nu}\left\{\left\langle\hat{\gamma} \frac{d}{d t}\left(f\left(E_{1}\right)-f\left(E_{2}\right)\right), \Psi_{\nu}(t)\right\rangle+\left\langle\hat{\gamma}\left(f\left(E_{1}\right)-f\left(E_{2}\right)\right), E(t)\right\rangle\right\} d t \\
& =\int_{0}^{\nu} \frac{d}{d t}\left\langle\hat{\gamma}\left(f\left(E_{1}\right)-f\left(E_{2}\right)\right), \Psi_{\nu}(t)\right\rangle \\
& =-\left\langle\hat{\gamma}\left(f\left(E_{1}(0, \cdot)\right)-f\left(E_{2}(0, \cdot)\right), \Psi_{\nu}(0)\right)\right\rangle=0,
\end{aligned}
$$

we obtain from (3.39) that

$$
\begin{aligned}
& \frac{1}{2}\|E(\nu)\|^{2}+\frac{1}{2} \sigma_{1}\left(\Psi_{\nu}(0), \Psi_{\nu}(0)\right)+\frac{1}{2} k\left\langle\Psi_{\nu}(0), \Psi_{\nu}(0)\right\rangle+\int_{0}^{\nu}\left\{\langle\gamma E(t), E(t)\rangle+c E(t, 0)^{2}\right. \\
& \left.-\operatorname{Re}\left\langle\int_{0}^{t} \alpha(t-s, \cdot) E(s, \cdot) d s, \Psi_{\nu}(t)\right\rangle\right\} d t-\operatorname{Re} \int_{0}^{\nu}\left\{\left\langle\beta\left(f\left(E_{1}\right)-f\left(E_{2}\right)\right), \Psi_{\nu}(t)\right\rangle\right. \\
& \left.-\left\langle\int_{0}^{t} \alpha(t-s, \cdot)\left(f\left(E_{1}(s, \cdot)\right)-f\left(E_{2}(s, \cdot)\right)\right) d s, \Psi_{\nu}(t)\right\rangle+\left\langle\hat{\gamma}\left(f\left(E_{1}\right)-f\left(E_{2}\right)\right), E\right\rangle\right\} d t=0 .
\end{aligned}
$$

Now

$$
\begin{aligned}
& \frac{1}{2}\|E(\nu)\|^{2}+\frac{1}{2} \sigma_{1}\left(\Psi_{\nu}(0), \Psi_{\nu}(0)\right) \leq \frac{1}{2}\left|\left\langle k \Psi_{\nu}(0), \Psi_{\nu}(0)\right\rangle\right|+\int_{0}^{\nu}\{|\langle\gamma E(t), E(t)\rangle| \\
& +\left|\left\langle\int_{0}^{t} \alpha(t-s, \cdot) E(s, \cdot) d s, \Psi_{\nu}(t)\right\rangle\right|+\left|\left\langle\beta\left(f\left(E_{1}\right)-f\left(E_{2}\right)\right), \Psi_{\nu}(t)\right\rangle\right| \\
& \left.+\left|\left\langle\int_{0}^{t} \alpha(t-s, \cdot)\left(f\left(E_{1}(s, \cdot)\right)-f\left(E_{2}(s, \cdot)\right)\right) d s, \Psi_{\nu}(t)\right\rangle\right|+\left|\left\langle\hat{\gamma}\left(f\left(E_{1}\right)-f\left(E_{2}\right)\right), E\right\rangle\right|\right\} d t
\end{aligned}
$$

From the definition of $\Psi_{\nu}$ we have that for each $t \in[0, T]$

$$
\left\|\Psi_{\nu}(t)\right\|^{2} \leq\left(\int_{0}^{\nu}\|E(\xi)\| d \xi\right)^{2} \leq T \int_{0}^{\nu}\|E(\xi)\|^{2} d \xi
$$

so that

$$
\left|\left\langle k \Psi_{\nu}(0), \Psi_{\nu}(0)\right\rangle\right| \leq k\left\|\Psi_{\nu}(0)\right\|^{2}=k T \int_{0}^{\nu}\|E(\xi)\|^{2} d \xi
$$

We use (3.47) and arguments similar to those behind the estimate (2.16) to obtain

$$
\begin{aligned}
& \left|\left\langle\int_{0}^{t} \alpha(t-s, \cdot) E(s, \cdot) d s, \Psi_{\nu}(t)\right\rangle\right| \leq\left(\int_{0}^{t} L_{3}\|E(\tau)\| d \tau\right)\left\|\Psi_{\nu}(t)\right\| \\
& \leq \frac{1}{2}\left(\int_{0}^{t} L_{3}\|E(\tau)\| d \tau\right)^{2}+\frac{1}{2}\left\|\Psi_{\nu}(t)\right\|^{2} \leq K_{1} \int_{0}^{\nu}\|E(\xi)\|^{2} d \xi
\end{aligned}
$$


for all $t \leq \nu$, where $K_{1}=\frac{1}{2}\left(L_{3}^{2} T+T\right)$. Next we estimate the terms in (3.45) containing the nonlinear function $f$ using the local Lipschitz property of $f$ and the fact that $E_{1}, E_{2}$ are uniformly bounded, i.e., in $L^{\infty}(0, T ; V)$. Thus for $t \leq \nu$

$$
\begin{aligned}
& \left|\left\langle\beta\left(f\left(E_{1}(t)\right)-f\left(E_{2}(t)\right)\right), \Psi_{\nu}(t)\right\rangle\right| \leq L_{1} K\|E(t)\|\left\|\Psi_{\nu}(t)\right\| \\
& \leq \frac{1}{2} L_{1}^{2} K^{2}\|E(t)\|^{2}+\frac{1}{2}\left\|\Psi_{\nu}(t)\right\|^{2} \\
& \leq \frac{1}{2} L_{1}^{2} K^{2}\|E(t)\|^{2}+\frac{T}{2} \int_{0}^{\nu}\|E(\xi)\|^{2} d \xi
\end{aligned}
$$

and

$$
\begin{aligned}
& \left|\left\langle\int_{0}^{t} \alpha(t-s, \cdot)\left(f\left(E_{1}(s, \cdot)\right)-f\left(E_{2}(s, \cdot)\right)\right) d s, \Psi_{\nu}(t)\right\rangle\right| \leq L_{3} K \int_{0}^{t}\|E(s)\| d s\left\|\Psi_{\nu}(t)\right\| \\
& \leq K_{2} \int_{0}^{\nu} \| E\left(\xi \|^{2} d \xi\right.
\end{aligned}
$$

where $K_{2}=\frac{1}{2}\left(L_{3}^{2} K^{2} T+T\right)$. We also have that

$$
\int_{0}^{\nu}\left|\left\langle\hat{\gamma}\left(f\left(E_{1}(t)\right)-f\left(E_{2}(t)\right)\right), E(t)\right\rangle\right| d t \leq \int_{0}^{\nu} L_{2} K\|E(t)\|^{2} d t .
$$

Using the estimates (3.47)-(3.51) in (3.45) we find that for $T \geq \nu \geq t$

$$
\frac{1}{2}\|E(\nu)\|^{2} \leq C \int_{0}^{\nu}\|E(t)\|^{2} d t
$$

where $\mathrm{C}$ is a constant. By the Gronwall inequality this yields that $E(\xi) \equiv 0$ on $(0, \mathrm{~T})$, and thus the solution of (2.8)-(2.9) is unique.

\section{Continuous Dependence}

In this section we show that the weak solution of the system (2.8)-(2.9) depends continuously on initial conditions and the source term. More precisely, we prove that the mapping $(\Phi, \Psi, \mathcal{J}) \rightarrow(E, \dot{E})$ is continuous from $V \times H \times H^{1}\left(0, T ; V^{*}\right)$ to $C(0, T ; H) \times L_{\text {weak }}^{2}(0, T ; H)$. To this end let us fix $(\Phi, \Psi, \mathcal{J}) \in V \times H \times H^{1}\left(0, T ; V^{*}\right)$ and consider sequences $\Phi^{n} \rightarrow \Phi$ in $V, \Psi^{n} \rightarrow \Psi$ in $H$ and $\mathcal{J}^{n} \rightarrow \mathcal{J}$ in $H^{1}\left(0, T ; V^{*}\right)$. We denote the unique weak solution corresponding to data $\left(\Phi^{n}, \Psi^{n}, \mathcal{J}^{n}\right)$ by $\left(E^{n}, \dot{E}^{n}\right)$ for each n. Our goal is to show that $E^{n} \rightarrow E$ in $C(0, T ; H)$ and $\dot{E}^{n} \rightarrow \dot{E}$ weakly in $L^{2}(0, T ; H)$ and $(E, \dot{E})$ satisfies $(2.8)$ with data $(\Phi, \Psi, \mathcal{J})$.

We first note that from the a priori estimate (2.22) we can conclude that the Galerkin approximations $\left\{E_{m}\right\}$ also satisfy

$$
\left\|\dot{E}_{m}\right\|_{L^{2}(0, T ; H)}^{2}+\nu\left\|E_{m}\right\|_{L^{2}(0, T ; V)}^{2}+2 c T\left\|\dot{E}_{m}(\cdot, 0)\right\|_{L^{2}(0, T)}^{2} \leq C \cdot T,
$$

for each $\mathrm{m}$, where the constant $\mathrm{C}$ is given as before and depends continuously on $\Phi, \Psi, \mathcal{J}$. Since we established the convergences $E_{m} \rightarrow E, \quad \dot{E}_{m} \rightarrow \dot{E}, \quad \dot{E}_{m}(\cdot, 0) \rightarrow E(\cdot, 0)$ weakly in 
$L^{2}(0, T ; V), L^{2}(0, T ; H)$ and $L^{2}(0, T)$, respectively, by the weak lower semicontinuity of the norms we obtain that $E$, the unique weak solution obtained in Section 1 also satisfies the inequality above, i.e.,

$$
\|\dot{E}\|_{L^{2}(0, T ; H)}^{2}+\nu\|E\|_{L^{2}(0, T ; V)}^{2}+2 c T\|\dot{E}(\cdot, 0)\|_{L^{2}(0, T)}^{2} \leq C \cdot T .
$$

Now if we consider the weak solution $E^{n}$ corresponding to data $\left(\Phi^{n}, \Psi^{n}, \mathcal{J}^{n}\right)$ it satisfies the same inequality (4.54) with $C^{n}=C^{n}\left(T, f, g, \Phi^{n}, \Psi^{n}, \mathcal{J}^{n}\right)$ instead of $C$. However, since $\left(\Phi^{n}, \Psi^{n}, \mathcal{J}^{n}\right) \rightarrow(\Phi, \Psi, \mathcal{J})$, there exists $\tilde{C}=\tilde{C}(T, f, g)$ independent of $\mathrm{n}$ such that $C^{n} \leq \tilde{C}$ for each $n$. Thus

$$
\begin{aligned}
& \left\{E^{n}\right\} \text { is bounded in } L^{2}(0, T ; V) \\
& \left\{\dot{E}^{n}\right\} \text { is bounded in } L^{2}(0, T ; H) \text { and } \\
& \left\{\dot{E}^{n}(\cdot, 0)\right\} \text { is bounded in } L^{2}(0, T) .
\end{aligned}
$$

We also know that $E^{n} \in C(0, T ; H)$ for all n. As in the proof of existence we can conclude that there exists $E \in L^{2}(0, T ; V)$ such that for a suitable subsequence (again denoted by $E^{n}$ )

$$
\begin{aligned}
E^{n} & \rightarrow E \text { weakly in } L^{2}(0, T ; V) \\
\dot{E}^{n} & \rightarrow \dot{E} \text { weakly in } L^{2}(0, T ; H) \\
\dot{E}^{n}(\cdot, 0) & \rightarrow E(\cdot, 0) \text { weakly in } L^{2}(0, T) .
\end{aligned}
$$

We can now proceed and finish the proof of the continuous dependence of weak solutions exactly as we did in the existence proof if we can show the stronger convergence

$$
E^{n} \rightarrow E \text { in } C(0, T ; H) .
$$

Note that no other property of the Galerkin approximations was used in the previous proof except these four convergences and the fact that $E_{m}$ satisfies (2.33). We establish the convergence $E^{n} \rightarrow E$ in $C(0, T ; H)$ using the Arzela-Ascoli theorem again. Following the arguments at the end of Section 2 and using the weak lower semicontinuity of norms we can argue that for the weak solutions corresponding to data $\left(\Phi^{n}, \Psi^{n}, \mathcal{J}^{n}\right)$, we have

$$
\left\|E^{n}(t)\right\|_{V}^{2} \leq \frac{1}{\nu} C^{n} \leq \frac{1}{\nu} \tilde{C} .
$$

Hence, we can conclude that $\left\{E^{n}(t)\right\}$ is bounded in V.

We can also see that $\left\{E^{n}\right\}$ is equicontinuous in $C(0, T ; H)$ :

$$
\begin{aligned}
& \left\|E^{n}(t+\Delta t)-E^{n}(t)\right\|^{2}=\left\|\int_{t}^{t+\Delta t} \dot{E}^{n}(\tau) d \tau\right\|^{2} \leq\left(\int_{t}^{t+\Delta t}\left\|\dot{E}^{n}(\tau)\right\| d \tau\right)^{2} \\
& \leq \Delta t \int_{t}^{t+\Delta t}\left\|\dot{E}^{n}(\tau)\right\|^{2} d \tau \leq \Delta t \tilde{C} .
\end{aligned}
$$

By an application of the Arzela-Ascoli theorem we can show that $\left\{E^{n}\right\}$ is relatively compact in $C(0, T ; H)$, so it has a subsequence (again denoted by $E^{n}$ ) that converges to $E$ in $C(0, T ; H)$. Indeed, this argument reveals that any subsequence has in turn a subsequence converging to the unique limit $E$ and hence the original sequence converges to $E$ in $C(0, T ; H)$. Now the proof of continuous dependence can be finished in the same fashion as the existence proof. 


\section{Application to electromagnetics}

The theoretical results in this paper are an initial effort in developing a foundation for nonlinear electromagnetics and the needed computational methods for such. The potential applications of nonlinear optics with light as an information carrier are widespread and are the basis of optical information technology and the use of optical fibers. They are of increasing importance with the development of materials (e.g., GaAs, InP, $\mathrm{KNbO}_{3}$ ) which possess outstanding nonlinear optical and electro-optical properties [7]. Our interests arise from the potential of using interrogating input pulses in the IR range (terahertz) in imaging and more specifically in detection algorithms.

Nonlinear optical effects are usually described through nonlinear polarization laws [6] and many of these laws can be formulated in the framework of this paper where we treat rather general nonlinearities. These include locally polynomial nonlinearities (e.g., so-called third order and higher effects $[6,7])$ that are bounded as the magnitude of the $E$ field saturates polarization mechanisms (e.g., the material "freezes" dielectrically at high field values). The assumption that the nonlinearity is increasing with the magnitude of the $E$ field is again a realistic one.

Though our treatment is for full wave propagation (as opposed to the popular paraxial approximate models [6] found widely in the literature), even here there are some tacit assumptions that may result in limiting approximations to phenomena that actually occur in nonlinear materials. Specifically, the one-dimensional model formulated in Section 2 depends on the tacit assumption that the polarization field $\bar{P}$ in the dielectric remains parallel to the electric field $\bar{E}$. Even then, the usual Maxwell equation $\nabla \cdot \bar{D}=0$ along with the constitutive law $\bar{D}=\epsilon_{0} \epsilon_{r} \bar{E}+f_{1}(P) \bar{P}$ need not result in $\nabla \cdot \bar{E}=0$. This is important in deriving the second order form of Maxwell's equation where the identity $\nabla \times \nabla \times \bar{E}=\nabla(\nabla \cdot \bar{E})-\nabla^{2} \bar{E}$ results in the simple Laplacian only if $\nabla \cdot \bar{E}=0$ or one assumes this term is negligible as often done in nonlinear optics ([6] p.54-60). Even with some obvious inadequacies, the model considered in this paper does facilitate treatment of a number of mechanisms of interest, including Debye

and Lorentz materials that are driven by nonlinear $E$ field inputs, e.g., $\dot{P}+\frac{1}{\tau} P=f(E)$.

\section{Acknowledgment}

The authors are grateful to Dr. Richard Albanese for numerous technical discussions, suggestions and encouragement to pursue efforts in nonlinear electromagnetics. This research was supported in part by the US Air Force Office of Scientific Research under grant AFOSRF49620-1-00-0026.

\section{References}

[1] H.T. Banks, M.W. Buksas and T. Lin, Electromagnetic Material Interrogation Using Conductive Interfaces and Acoustic Wavefronts, SIAM Frontiers in Applied Mathematics, Philadelphia, 2000. 
[2] H.T. Banks, D.S. Gilliam and V.I. Shubov, 'Global solvability for damped abstract nonlinear hyperbolic systems', Differential and Integral Equations, 10 (1997), 309332.

[3] H. T. Banks, K. Ito and Y. Wang, 'Well-posedness for damped second order systems with unbounded input operators', CRSC-TR93-10, NCSU, June 1993; Differential and Integral Equations Vol. 8 (1995), 587-606.

[4] H.T. Banks, R.C. Smith and Y. Wang, Smart Material Structures, Modeling, Estimation and Control, Masson/Wiley, Paris/Chichester, 1996.

[5] F. Bloom, 'Asymptotic bounds for solutions to a system of damped integrodifferential equations of electromagnetic theory,' J. Math. Anal. Appl., Vol. 73, (1980), 524-542.

[6] R.W. Boyd, Nonlinear Optics, Academic Press, San Diego, 1992.

[7] P. Günter (ed.), Nonlinear Optical Effects and Materials, Springer, Berlin, 2000.

[8] I. Kurbanov, 'Solvability of the linear Maxwell equations with memory', Ukr. Mat. J., Vol. 43, (1991), 619-635.

[9] J.L. Lions, Optimal Control of Systems Governed by Partial Differential Equations, Springer-Verlag, New York, 1971.

[10] A. Milani and R. Picard, 'Evolution equations with nonlinear constitutive laws and memory effects', Differential and Integral Equations, Vol. 15 (2002), 327-344.

[11] D.L. Mills, Nonlinear Optics, Springer-Verlag, Berlin, 1991.

[12] A.W. Naylor and G.R. Sell, Linear Operator Theory in Engineering and Science, Springer Verlag, New York, 1982.

[13] A.C. Newell and J.V. Moloney, Nonlinear Optics, Addison-Wesley, Redwood City, 1991.

[14] J. Prüss, Evolutionary Integral Equations and Applications, Birkhäuser, Basel, 1993.

[15] Y.R. Shen Principles of Nonlinear Optics, Wiley, New York, 1984.

[16] C. Sulem and P-L. Sulem, The Nonlinear Schrödinger Equation, Self-Focusing and Wave Collapse, Springer-Verlag, New York, 1999.

[17] Wloka, J., Partial Differential Equations, Cambridge Univ. Press. 1992. 